\title{
Genetic basis for childhood interstitial lung disease among Japanease infants and children
}

\author{
Itaru Hayasaka ${ }^{1,5}$, Kazutoshi Cho ${ }^{1,5}$, Takuma Akimoto ${ }^{1,5}$, Masahiko Ikeda ${ }^{1,5}$, Yutaka Uzuki ${ }^{1,5}$, Masafumi Yamada ${ }^{2,5}$, \\ Koh Nakata ${ }^{3,5}$, Itsuko Furuta ${ }^{4,5}$, Tadashi Ariga ${ }^{2,5}$ and Hisanori Minakami ${ }^{4,5}$
}

BACKGROUND: Genetic variants responsible for childhood interstitial lung disease (chILD) have not been studied extensively in Japanese patients.

METHODS: The study population consisted of 62 Japanese chILD patients. Twenty-one and four patients had pulmonary hypertension resistant to treatment $(\mathrm{PH})$ and hypothyroidism, respectively. Analyses of genetic variants were performed in all 62 patients for SFTPC and $A B C A 3$, in all $21 \mathrm{PH}$ patients for FOXF1, and in a limited number of patients for NKX2.1.

RESULTS: Causative genetic variants for chILD were identified in $11(18 \%)$ patients: SFTPC variants in six, NKX2.1 variants in three, and FOXF1 variants in two patients. No patients had $A B C A 3$ variants. All three and two patients with NKX2.1 variants had hypothyroidism and developmental delay, respectively. We found six novel variants in this study.

CONCLUSION: Mutations in SFTPC, NKX2.1, and FOXF1 were identified among Japanese infants and children with chILD, whereas $A B C A 3$ mutations were rare.

$\mathbf{R}$ espiratory difficulties are often seen in neonates because of poor adaptation to lung respiration. However, some cases of respiratory failure cannot be explained by prematurity or poor adaptation. Childhood interstitial lung disease (chILD) is one such rare pathology that causes respiratory dysfunction in infants and children (1).

chILD comprises a group of disorders that cause respiratory dysfunction in infants and children. The chILD disorders include pulmonary alveolar proteinosis (PAP) diagnosed based on bronchoalveolar lavage (BAL) and/or lung histology (2), interstitial pneumonitis (IP) diagnosed based on lung histology, and alveolar capillary dysplasia with misalignment of pulmonary veins (ACDMPV) diagnosed based on lung histology (3). Widespread ground glass opacification and individually irregular consolidation on the dependent side or geographic opacification on high-resolution computed tomography are helpful for suspicion of chILD (4).
Some cases of chILD have genetic abnormalities, and hereditary chILD usually occurs in early childhood (5). Known genetic abnormalities include mutations in $S F T P B$ for surfactant protein (SP)-B deficiency (6), SFTPC for SP-C abnormality (7), $A B C A 3$ for $A B C A 3$ deficiency (8), and NKX2.1 for TTF-1 dysfunction among infants with PAP or IP, and FOXF1 among infants with ACDMPV $(9,10)$. In addition, known abnormalities responsible for PAP include abnormalities in granulocyte macrophage colony-stimulating factor (GM-CSF) receptor $(11,12)$ and the presence of antibodies against GM-CSF (13). Therefore, assessment of GM-CSFstimulating phosphorylation of signal transducer and activator of transcription-5 (pSTAT-5) (12) and/or determination of anti-GM-CSF antibody are also helpful to understand the pathogenesis of PAP.

We launched a system to aid Japanese neonatologists and pediatricians to search for genetic causes of unexplained respiratory failure in 2011, and the results of our study in 43 cases during the period between February 2011 and July 2013 were reported previously (14). Here, we report the results of the analyses of genetic abnormalities in an additional 62 infants and children with respiratory failure between August 2013 and June 2016.

\section{METHODS}

The present system was announced to Japanese neonatologists/ pediatricians via the E-mail Network of Neonatologists in February 2011 after receiving approval from the Institutional Review Board of the Faculty of Medicine and Graduate School of Medicine, Hokkaido University. Collaboration with the Japan Society for Neonatal Health and Development, formerly the Japan Society for Premature and Newborn Medicine (JSPNM), was begun in February 2012 to facilitate collection of cases with severe and unexplained lung dysfunction: the JSPNM announced 3,300 neonatologists four times annually since February 2012 to prospectively register patients with unexplained sustained respiratory distress due to genetic disorders and of unknown origin. Patients with respiratory failure due to known reasons were excluded. Most Japanese neonatologists also worked as professional pediatricians to treat school-aged children with lung dysfunction, were members of the JSPNM, and were working at $\sim 90 \%$ of all facilities with neonatal intensive care units in Japan.

\footnotetext{
${ }^{1}$ Maternity and Perinatal Care Center, Hokkaido University Hospital, Sapporo, Japan; ${ }^{2}$ Department of Pediatrics, Faculty of Medicine and Graduate School of Medicine, Hokkadio University, Sapporo, Japan; ${ }^{3}$ Bioscience Medical Research Center, Niigata University Medical and Dental Hospital, Niigata, Japan; ${ }^{4}$ Department of Obstetrics, Faculty of Medicine and Graduate School of Medicine, Hokkadio University, Sapporo, Japan. Correspondence: Kazutoshi Cho (chotarou@med.hokudai.ac.jp)

${ }^{5}$ These authors contributed equally to this work.

Received 19 November 2016; accepted 2 September 2017; advance online publication 1 November 2017. doi:10.1038/pr.2017.217
} 


\section{Patients}

The inclusion criteria for study entry were as follows: $\leq 10$ years of age at onset, severe sustained ( $>1$ week) lung dysfunction, diffused pulmonary infiltrate on chest X-ray, and/or ground glass opacification on computed tomography. Patients with known causative factors, such as infection, congenital heart disease, systemic bone disease, neuromuscular disease, malformations, pulmonary hypertension after birth asphyxia, or bronchopulmonary dysplasia associated with prematurity, were excluded. Sixty-two patients with chILD were analyzed in this study. All the 62 patients were referred to us for analysis of genetic variants in the 35 -month period between August 2013 and June 2016. All the 62 families of the 62 patients provided detailed clinical information and blood samples for genetic analyses with written informed consent. These 62 patients were admitted at 48 hospitals located widely throughout Japan. Pulmonary hypertension resistant to treatment $(\mathrm{PH})$ was defined as that evidenced on echocardiography and are resistant to treatment, including sedation, diuretics, oxygen administration, and vasodilators, such as inhaled nitric oxide gas and prostaglandin $\mathrm{I}_{2}$ in this study. Two patients with $\mathrm{PH}$ were treated with extracorporeal membrane oxygenation.

\section{Analyses of the SFTPC, ABCA3, FOXF1, and NKX2.1}

Genomic DNA was extracted from peripheral blood using a QIAamp DNA Blood Mini Kit (Qiagen, Hilden, Germany). PCR methods for $S F T P C, A B C A 3$, and FOXF1 were described previously (14). Analysis of NKX2.1 was introduced in February 2015. Purified products were subjected to nucleotide sequence analysis by a commercial sequencing service (FASMAC, Kanagawa, Japan). Nucleotide sequences were compared with the reported reference sequences: NM_003018.3 (SFTPC), NM_001089.2 (ABCA3), NM_001451.2 (FOXF1), and NM_001079668.2 (NKX2.1). Analyses of SFTPC and ABCA3 were performed in all patients. Analysis of NKX2.1 was performed in a limited number of patients referred to us on or after February 2015. Analysis of FOXF1 was performed in all patients with PH. No patients showed clinical features compatible with SP-B deficiency, recurrent respiratory distress syndrome (RDS) at birth, and following PAP. As SP-B deficiency has been considered to be rare in Japanese chILD (14), SFTPB analysis was not performed in any patient in this study. Although a patient with ABCA3 deficiency also shows recurrent $\mathrm{RDS}$, there was no information about prevalence of ABCA3 deficiency in Japan; therefore, we performed ABCA3 analysis for all patients. Candidate missense variants were evaluated with SIFT (http://sift.jcvi.org), PolyPhen-2 (http://genetics.bwh. harvard.edu/pph2/), and MutationTaster (http://www.mutationta ster.org). Moreover, allele frequencies of the variants were checked in East Asians according to the 1000 Genomes and ExAC/gnomAD.

\section{Analyses of GM-CSF-Induced PSTAT-5 and Anti-GM-CSF Antibody}

Peripheral blood mononuclear cells were suspended in RPMI 10\% fetal bovine serum at a concentration of $1 \times 10^{6}$ cells $/ \mathrm{ml}$, and incubated in the presence or absence of $20 \mathrm{ng} / \mathrm{ml}$ GM-CSF for $15 \mathrm{~min}$. Whole-cell lysates were prepared by homogenization in $1 \times$ sodium dodecyl sulfate (SDS) sample buffer, separated by glycine/ SDS-polyacrylamide gel electrophoresis according to the standard procedures, and transferred onto PVDF membranes. Anti-STAT-5 (pY694) antibody (BD, San Diego, CA) was used at a final concentration of $0.5 \mu \mathrm{g} / \mathrm{ml}$, and anti-actin antibody (Sigma, St Louis, $\mathrm{MO}$ ) was used at a final concentration of $1 \mu \mathrm{g} / \mathrm{ml}$ as a loading control. Serum GM-CSF auto-antibody was measured using the method described previously (15) by one of the authors (K.N.) at Niigata University Medical and Dental Hospital.

\section{RESULTS}

Lung histology was examined in 14 of the patients (Figure 1). Lung specimens were reviewed at the individual institutions and we also confirmed the findings. Other patients of ILD

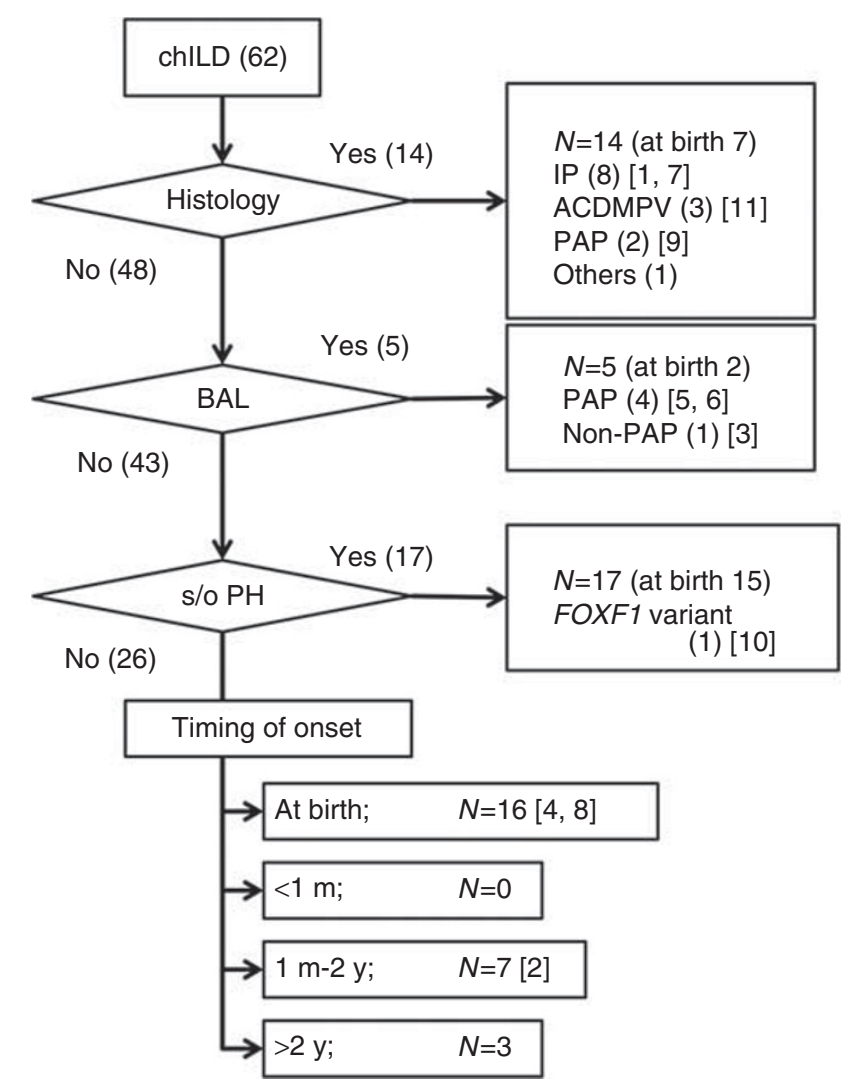

Figure 1. Process of the study. Numbers in square brackets refer to subjects listed in Table 2, and numbers in round brackets refer to those of patients. Histology and BAL were available in 14 and 5 patients, respectively. IP was diagnosed in eight patients by histology, and PAP was diagnosed in six patients (in two patients by histology and in four patients by BAL). Seventeen infants with $\mathrm{PH}$ were clinically suspected of $\mathrm{PH}$ and received FOXF1 analysis. FOXF1 variant was detected in a patient. Among the remaining 26 patients, the SFTPC variant was detected in patient onset at 1.5 years, and SFTPC and NKX2.1 variants were detected in patients with onset at birth. BAL, bronchoalveolar lavage; IP, interstitial pneumonitis; PAP, pulmonary alveolar proteinosis.

were diagnosed from clinical features. Eight patients were diagnosed with IP, seven had nonspecific interstitial pneumonia, and one had desquamative interstitial pneumonia. Pathological diagnoses were ACDMPV in three, PAP in two, and mild nonspecific hypertrophy of vascular media in one. Five patients underwent BAL and four were diagnosed with PAP based on typical gross appearance and microscopic findings, with milky white color and massive precipitate, PAS-positive proteinous material, respectively. Another patient who received BAL was provisionally diagnosed with IP and clinically diagnosed with chILD without BAL findings compatible with PAP. Thus, a total of 19 patients had diagnoses based on histology/BAL. Of the 62 study subjects, 21 exhibited $\mathrm{PH}$ (Table 1). Thyroid status was known in all the 62 patients, and hypothyroidisms of four patients were diagnosed at the neonatal period. The median (range) age at DNA sampling was 2 months (0d-16y). 
Table 1. Backgrounds of the 62 study subjects

\begin{tabular}{|c|c|}
\hline Available lung histology & $14(23 \%)$ \\
\hline Histology-proven IP & $8(13 \%)$ \\
\hline Histology-proven PAP & $2(3.2 \%)$ \\
\hline Histology-proven ACDMPV & $3(4.8 \%)$ \\
\hline Nonspecific pulmonary hypertension & $1(1.6 \%)$ \\
\hline Available BAL & $5(8.1 \%)$ \\
\hline BAL-proven PAP & $4(6.5 \%)$ \\
\hline IP-suspected & $1(1.6 \%)$ \\
\hline \multicolumn{2}{|l|}{ Available thyroid status } \\
\hline Hypothyroidism & $4(6.5 \%)$ \\
\hline Pulmonary hypertension & $21(34 \%)$ \\
\hline Gestational age at birth (weeks) & $39(28-41)$ \\
\hline Age at onset $(d, m, y)$ & $0 d(0 d-9 y)$ \\
\hline At birth & $40(65 \%)$ \\
\hline$<1 \mathrm{~m}$ & $2(3.2 \%)$ \\
\hline $1 \mathrm{~m}-2 \mathrm{y}$ & $14(23 \%)$ \\
\hline$\leq 2.0 \mathrm{y}$ & $6(9.7 \%)$ \\
\hline Age at DNA sampling & $2 m(0 d-16 y)$ \\
\hline Mortality & $11(18 \%)$ \\
\hline SFTPC analysis performed & $62(100 \%)$ \\
\hline$A B C A 3$ analysis performed & $62(100 \%)$ \\
\hline NKX2.1 analysis performed & $38(61 \%)$ \\
\hline FOXF1 analysis performed & $21(34 \%)$ \\
\hline
\end{tabular}

ACDMPV, alveolar capillary dysplasia with misalignment of pulmonary vein; BAL, bronchoalveolar lavage; $d$, day; IP, interstitial pneumonitis; $m$, month; PAP, pulmonary alveolar proteinosis; $y$, year.

Data are presented as the median (range) or number of patients (\% of the starting cohort).

Profiles of 11 Patients with Detected Causative Genetic Variants Genetic variants considered responsible for the disease were detected in $11(18 \%)$ of the 62 patients (Table 2). The median (range) gestational week at birth was $40(36-41)$ and the median birthweight was $3.24(2.53-3.76) \mathrm{kg}$ in these 11 patients. Six patients had diagnoses based on lung histology/ BAL (Subjects 1, 5, 6, 7, 9, and 11). Thus, 32\% (6/19) of patients with diagnoses based on lung histology/BAL exhibited genetic variants. Of the nine patients with SFTPC or NKX2.1 variants, none had PH.

Among the nine genetic variants detected in the 11 patients (Subjects $1-11$ in Table 2), three (including SFTPC c. $218 \mathrm{~T}>$ C, p.Ile73Thr, SFTPC c.134 T $>$ G, p.Leu45Arg, and FOXF1 c.256C $>$ T, p.Arg86Trp) are known to be responsible for lung dysfunction (16-18). To our knowledge, six other mutations in six patients in the present study have not been reported previously.

\section{Analysis of SFTPC and ABCA3}

All the 62 patients underwent analyses of SFTPC and $A B C A 3$ genes. None exhibited $A B C A 3$ variants, but six exhibited SFTPC variants: c. $218 \mathrm{~T}>\mathrm{C}$, p.Ile73Thr in three (Subjects 1, 2 , and 3 in Table 2), and c.541delC, p.Leu181Trpfs ${ }^{\star} 5$ (Subject 4); c.134 T>G, p.Leu45Arg (Subject 5); and c.181 A>G, p. Ser61Gly (Subject 6) in one each.

Lung dysfunction occurred at ages ranging from 1 year 0 months to 1 year 7 months in our three patients with c. $218 \mathrm{~T}>\mathrm{C}$, p.Ile73Thr variant manifesting clinical features of IP. In three patients with other SFTPC variants (Subjects 4, 5, and 6), lung dysfunction occurred immediately after birth. Subject 4 with a novel mutation of c.541delC, p. Leu181Trpfs5X required mechanical ventilation, steroids, and home oxygen therapy until 1, 3, and 9 months of age, respectively. This patient required no supplemental oxygen when last seen at 6 years of age. At the age of 16 days, Subject 5 with c.134 T $>$ G, p.Leu45Arg mutation exhibited ground glass opacification on computed tomography, and was shown to have PAP with BAL at that age. This patient required home oxygen therapy and died from pulmonary aspiration at 2.4 years of age with a weight of $6.0 \mathrm{~kg}$ while awaiting lung transplantation. Subject 6 with a novel mutation of c.181 A > G, p.Ser61Gly was diagnosed with PAP at the age of 30 days, required mechanical ventilation until 1.3 years of age, and was treated with hydroxychloroquine and steroids, but died 4 months after lung transplantation at 9.3 years of age.

The asymptomatic parents of four patients (Subjects 1, 3, 4, and 5) underwent genetic testing: one SFTPC variant was derived from de novo mutation (Subject 5), and two (Subjects 1 and 4) and one (Subject 3) were inherited from the maternal and paternal family lines, respectively.

\section{Analysis of NKX2.1}

Analysis of the NKX2.1 gene was introduced in February 2015 , and was performed in 38 (61\%) of the 62 patients. Three patients exhibited NKX2.1 variants: c.1117C > T, p.Gln373X (Subject 7 in Table 2); c.1016_1017insCCATCTCCGTGGGC AGCGG, p.Gly339fs (Subject 8); and c.954_958GCAGG > CAG, p.Gln318fs (Subject 9) in one each. Thus, the frequency of NKX2.1 variants was $7.9 \%$ (3/38). All three patients with NKX2.1 variant had hypothyroidism (Subjects 7, 8, and 9). NKX2.1 analysis was performed in four patients with and in 34 without hypothyroidism, respectively. All three patients with NKX2.1 variants presented with cyanosis, and showed high blood levels of KL-6 (a protein expressed in lung epithelial cells (19); range, 5,467-10,000 U/ml), SP-A (33$118 \mathrm{ng} / \mathrm{ml})$, and SP-D $(111-397 \mathrm{ng} / \mathrm{ml})$, as well as ground glass opacification on pulmonary computed tomography. Treatment with steroids was ineffective in Subject 9 with PAP, but a combination of steroids and hydroxychloroquine was apparently effective in two patients (Subjects 7 and 8). Azathioprine was ineffective in Subject 7 and surfactant replacement was apparently effective in Subject 8. All three patients required home oxygen therapy. Total anomalous 
Table 2. Genetic variations detected in 11 patients

\begin{tabular}{|c|c|}
\hline $\begin{array}{l}\text { Subject, gender/BW } \\
\text { (percentile)/GA }\end{array}$ & Histo \\
\hline \multicolumn{2}{|l|}{ SFTPC variation } \\
\hline 1. Male/3.31 (60th)/41 & NSIP/no/no \\
\hline 2. Male/3.46 (80th)/40 & NA/no/no \\
\hline 3. Male/3.40 (70th)/41 & NA/no/no \\
\hline 4. Male/3.24 (33rd)/41 & NA/no/no \\
\hline 5. Female/2.53 (46th)/36 & $\mathrm{PAP} / \mathrm{no} / \mathrm{no}$ \\
\hline 6. Male/3.76 (99th)/39 & $\mathrm{PAP} / \mathrm{no} / \mathrm{no}$ \\
\hline
\end{tabular}

Onset/outcome

Genetic variation/Polyhen-2/origin

Treatment

Histology/PH/complication

Onset/outcome

1 y $0 \mathrm{~m} /$ survive $(2$ y $\quad$ c.218 T > C, p.lle73Thr/0.855/maternal $4 \mathrm{~m})$

1 y $5 \mathrm{~m}$ /survive $\quad$ c.218 T>C, p.lle73Thr/0.855/unknown (2y $8 \mathrm{~m})$

1 y $7 \mathrm{~m} /$ survive $(2$ y c.218 T > C, p.lle73Thr/0.855/paternal $5 \mathrm{~m})$

0d/survive $(6$ y) c.541delC, p.Leu181Trpfs*5/-/maternal

$0 \mathrm{~d} /$ death $(2$ y $5 \mathrm{~m}$ ) c.134 T>G, p.Leu45Arg/1.000/de novo

$0 \mathrm{~d} /$ death $(9 \mathrm{y})$

c.181 A>G, p.Ser61Gly/0.999/unknown

$11 \mathrm{~m} /$ survive (11 y) c.1117C > T, p.Gln373X/-/unknown

7. Female/2.68 (16th)/40 NSIP/no/hypothyroidism

8. Male/2.90 (54th)/38 NA/no/hypothyroidism, developmental delay

9. Female/3.65 (96th)/40 PAP/no/hypothyroidism,TAPVC, developmental delay

\section{$0 \mathrm{~d} /$ Survive $(1 \mathrm{y}$ \\ $6 \mathrm{~m})$ \\ c.1016_1017insCCATCTCCGTGGGCAGCGG, p. Gly339fs/-/de novo}

$3 \mathrm{~m} /$ survive $(2 \mathrm{y}$

$9 \mathrm{~m})$
c.954_958GCAGG > CAG, p.Gln318fs/-/de novo

\section{FOXF1 variation}

10. Female/2.70 (10th)/40 NA/yes/no

11. Female/2.99 (59th)/39 ACDMPV/yes/anal atresia

$0 \mathrm{~d} /$ survive $(4 \mathrm{~m}) \quad$ c.256C $>$ T, p.Arg86Trp/1.000/de novo

$0 \mathrm{~d} /$ death $(5 \mathrm{~d}) \quad$ c.852_856delTATCA, p.Tyr284X/-/unknown
PSL $2 \mathrm{mg} / \mathrm{kg} /$ day, $\mathrm{HCO}$, HOT

PSL $2 \mathrm{mg} / \mathrm{kg} /$ day, no supplemental oxygen at 2 y $8 \mathrm{~m}$

PSL $1 \mathrm{mg} / \mathrm{kg} /$ day, HCQ, HOT

Surfactant, PSL 2 mg/kg/day, no supplemental oxygen at $6 y$

Surfactant, HOT

Surfactant, mPSL, HCQ, HOT, lung transplantation

PSL $1.5 \mathrm{mg} / \mathrm{kg} /$ day, $\mathrm{HCQ}$, azathioprine levothyroxine, HOT

Surfactant, steroids, sivelestat, $\mathrm{HCQ}$, levothyroxine HOT

surgery for TAPVC, PSL 2 mg/kg/day, levothyroxine, HOT

Surfactant, milrinone, sildenafil, hospitalization Surfactant, colostomy for anal atresia, mPSL, milrinone

ACDMPV, alveolar capillary dysplasia with misalignment of pulmonary vein; BW, birthweight (kg); GA, gestational age at birth (weeks of gestation); HCQ, hydroxychloroquine; HOT, home oxygen therapy; mPSL, methylprednisolone pulse therapy; NA, not available; NSIP, nonspecific interstitial pneumonia; PAP, pulmonary alveolar proteinosis; PH, pulmonary hypertension; PSL, prednisolone; TAPVC, total anomalous pulmonary venous connection.

Survive and Death means survival to age and death at the age indicated in parenthesis, respectively. 
pulmonary venous connection (TAPVC) was corrected surgically in the neonatal period in Subject 9. These patients were followed at individual institutes and our information has been updated. The patient with hypothyroidism and ILD without an NKX2.1 was born with 38 weeks of gestation and $3,110 \mathrm{~g}$ of birthweight. Lung biopsy at 2 years showed nonspecific interstitial pneumonia. He had mild hyperactivity but no developmental delay at 3 years. He had chronic respiratory failure resistant to steroids, hydroxychloroquine, and clarithromycin.

The asymptomatic parents of two patients (Subjects 8 and 9) underwent genetic testing: both were derived from de novo mutation. Sequencing strategy in this study did not permit assessment of deletions including NKX2.1.

\section{Analysis of FOXF1}

Twenty-one patients with $\mathrm{PH}$, three with histologically proven ACDMPV, 17 clinically suspected of ACDMPV with infantile-onset $\mathrm{PH}$, and one with multiple anomalies underwent FOXF1 analyses and two exhibited FOXF1 variants (Figure 1 and Table 1): c.256C > T, p.Arg86Trp in one (Subject 10 without available lung histology in Table 2) and c.852_856delTATCA, p.Tyr284X in the other (Subject 11 with ACDMPV). Subject 10 had PH immediately after birth, was treated with mechanical ventilation, inhaled nitric oxide and prostaglandin $I_{2}$, and was still alive as an inpatient at 4 months of age. Subject 11 with a novel mutation of $\mathrm{p}$. Tyr284X had anal atresia, and died from $\mathrm{PH}$ at the age of 5 days despite aggressive treatment, including mechanical ventilation, inhaled nitric oxide, and prostaglandin $\mathrm{I}_{2}$; a diagnosis of ACDMPV was made based on analyses of an autopsied lung specimen. The asymptomatic parents of Subject 10 underwent genetic testing, but neither had c.256C $>$ T, p.Arg86Trp (Table 2). In two of the three patients with ACDMPV diagnosed based on lung histology in this study, FOXF1 variants were not detected. Sequencing strategy in this study did not permit assessment of deletions including and surrounding FOXF1.

\section{Assessment of pSTAT-5 and Anti-GM-CSF Antibody}

Three of the six patients diagnosed with PAP based on histology/BAL were shown to have genetic variants (Subjects 5, 6, and 9 in Table 2). None of the remaining three PAP patients had SFTPC, ABCA3, or NKX2.1 variants. Two PAP patients (Subject 9 with NKX2.1 variants and one without genetic variant) underwent assessment of pSTAT-5 and antiGM-CSF antibodies; the patient without genetic variant was shown to have significant anti-GM-CSF antibody titer in the blood. Thus, factors considered responsible for the disease were detected in four of the six patients diagnosed with PAP based on histology/PAP in this study. The two patients with neither genetic variants considered responsible for the disease nor abnormalities of pSTAT-5 or anti-GM-CSF antibody had clinical diagnoses other than PAP; primary immunodeficiency disease in one and juvenile idiopathic arthritis in the other. The latter patient was diagnosed with idiopathic arthritis at the age of 7 years, experienced recurrent pneumonia while on steroids with immunosuppressant, was diagnosed with PAP at 8 years of age based on lung histology, and was shown to have SFTPC c.115G $>$ T pVal39Leu in this study. This variant was judged as "damaging" on SIFT, "benign" on PolyPhen-2, and "disease-causing" on MutationTaster. However, SFTPC c.115G $>$ T, p.Val39Leu was considered unlikely to be the causative factor of PAP of this patient based on the allele frequency of SFTPC c.115G $>$ T, p.Val39Leu, i.e., 0.7 and $0.05 \%$, in East Asians according to the 1000 Genomes Project and ExAC/gnomAD, respectively.

\section{DISCUSSION}

The present system was helpful for determination of genetic variants considered responsible in $11(18 \%)$ of the 62 patients with chILD, and suggested that among Japanese chILD patients NKX2.1 variants are relatively common. Among the nine genetic variants detected in the 11 patients (Subjects 111 in Table 2), three (including SFTPC c.218 T $>$ C, p. Ile73Thr, SFTPC c.134 T>G, p.Leu45Arg, and FOXF1 c.256C $>\mathrm{T}$, p.Arg86Trp) are known to be responsible for lung dysfunction (16-18). To our knowledge, six other mutations in six patients in the present study have not been reported previously. These six variants were not listed in the East Asian database in 1000 Genome Project and ExAC/ gnomAD. However, it was speculated that these six mutations were responsible for the lung dysfunction of six patients based on followings. The pro-SP-C amino acids of codon 61 are well preserved in many mammals, and SFTPC c.181 A>G, p. Ser61Gly in Subject 6 was judged as "damaging" with SIFT, "probably damaging (0.999)" with PolyPhen-2, and "diseasecausing" with MutationTaster. Frameshift mutation can cause abnormal protein function, and the frameshift mutation, SFTPC p.His142fs, is reported in a neonate with SP-C abnormality (20). Frameshift mutations were detected in three cases in this study: SFTPC p.Leu181Trpfs ${ }^{\star} 5$ (Subject 4), NKX2.1 p.Gly339fs (Subject 8), and NKX2.1 p.Gln318fs (Subject 9). Nonsense mutation can cause abnormal protein function, and those of NKX2.1 p.Gln373X (Subject 7) and FOXF1 p.Tyr284X (Subject 11) were detected in two patients in the present study. Thus, a "damaging" mutation in Subject 6, frameshift mutations in Subjects 4, 8, and 9, and nonsense mutations in Subjects 7 and 11 were considered responsible for lung dysfunction in these six infants.

Of the SFTPC variants detected in this study, c.218 T > C, p. Ile73Thr detected in three patients (Subjects 1-3) accounts for more than 25\% of patients with SFTPC variants presenting with clinical features of both IP and PAP (21), and varying age at onset (22).

FOXF1 variants were detected in two patients in this study (Subjects 10 and 11). As the variant of p.Arg86Trp detected in Subject 10 can cause ACDMPV (18), Subject 10 may have suffered from ACDMPV. However, this patient lacked anomalies in the heart, alimentary tract, and urogenital organs, although $\sim 80 \%$ of ACDMPV patients have anomalies of other organs, particularly of the cardiovascular, 


\section{Articles | Hayasaka and Akimoto}

gastrointestinal, and/or genitourinary systems (23). The results of a previous study substantiated the suggestion that mutations in FOXF1 led to manifestation of ACDMPV and that this transcription factor is involved in the development of the pulmonary, cardiovascular, gastrointestinal, and genitourinary systems (18). Although rare cases of histologically proven ACDMPV with FOXF1 variants showed slow onset and could survive with intensive care (24), with affected patients typically developing lung dysfunction and $\mathrm{PH}$ a few hours after birth, consistent with the findings in two of our patients (Subjects 10 and 11).

FOXF1 mutation was not detected in two of three patients diagnosed with ACDMPV based on lung histology in this study. We sequenced only the coding region of FOXF1 in this study. As variants in upstream regions and copy number variants of FOXF1 can cause ACDMPV (25), the possibility of these abnormalities in the two patients without FOXF1 mutation in our setting could not be excluded.

Three patients had NKX2.1 mutations (Subjects 7, 8, and 9); none of these mutations (c.1117C $>$ T, p.Gln373X, c.1016_1017insCCATCTCCGTGGGCAGCGG, p.Gly339fs, and c.954_958GCAGG > CAG, p.Gln318fs) has been reported previously. All three patients with NKX2.1 mutations had hypothyroidism. Similar to previous findings, NKX2.1 variants were more common among those with hypothyroidism. TTF-1 encoded by NKX2.1 is a protein expressed in the thyroid gland, lung primordium, and central nervous system, and is expressed specifically in the epithelial cells of the lung (26). TTF-1 abnormality caused by NKX2.1 mutation was first reported in 1998 (ref. 27) and is associated with lung dysfunction, hypothyroidism, chorea, and/or psychomotor developmental delay (28). Lung dysfunction, hypothyroidism, and abnormalities in the central nervous system are seen in 54,87 , and $93 \%$ of patients with NKX2.1 mutations, respectively, with all three seen in $50 \%$ of patients, and both hypothyroidism and central nervous system abnormalities are seen in $30 \%$ of patients (29). Subject 9 had TAPVC in this study. However, to our knowledge, there have been no literature reports describing TAPVC in patients with NKX2.1 mutation. The finding of TAPVC was associated with the finding of the mutation, the question is whether it was causally related is what is unknown. Thirty-five (92\%) of the thirty-eight patients examined did not show NKX2.1 mutation in this study. We sequenced only the coding region of NKX2.1 in this study, and this may explain the NKX2.1 mutation detection rate of $8 \%(3 / 38)$ in this study.

To our knowledge, a total of 17 Japanese cases of NKX2.1 mutations have been previously described in seven reports to date (30-36). These 17 cases showed various abnormalities: chorea in 14 (82\%), hypothyroidism in 11 (65\%), recurrent respiratory infections in six $(35 \%)$, and mental retardation in four (24\%). However, none of the 17 cases exhibited RDS, although lung dysfunction associated with NKX2.1 mutations includes RDS other than IP, PAP, and recurrent respiratory infections; Hamvas et al. reported symptoms suggestive of RDS, chILD, and recurrent respiratory infections in $76 \%$,
$19 \%$, and $43 \%$ of patients, respectively (9). As our system was developed to aid in the determination of genetic variants for chILD patients, it was not clear how many Japanese infants/ children with symptoms suggestive of RDS and recurrent respiratory infections had NKX2.1 mutations.

In this study, genetic abnormalities considered causative of lung dysfunction were found in three of six patients with PAP diagnosed based on histology/BAL. In our previous study (14), genetic variants considered causative of lung dysfunction was detected in all three patients with PAP diagnosed based on histology/BAL (two SFTPC mutations and one ABCA3 mutation). Thus, our system indicated that the majority of Japanese patients with infantile and childhood PAP had mutations in SFTPC, ABCA3, or NKX2.1, and suggested that investigation of these abnormalities can efficiently detect causative genetic abnormalities.

None of the 62 and only one of 43 infants tested had an $A B C A 3$ mutation in the present and our previous studies (14), respectively, while six of the $62(9.7 \%)$ and four of the 43 infants (9.3\%) tested had SFTPC mutations in the present and our previous studies (14), respectively. Thus, ABCA3 deficiency was suggested to be rare in the Japanese population. However, ABCA3 deficiency is relatively common in patients with SP dysfunction disorders in Western countries $(37,38)$. These results suggested that there are ethnic differences in the prevalence rates of ABCA3 deficiency. The allele frequency of Glu292Val, the most common variant of $A B C A 3$ in Europeans, is 4/1,000 according to the European database, whereas it is $0 / 1,000$ according to the East Asian database in 1000 Genome Project. This may explain the low frequency of $A B C A 3$ abnormalities in our study population.

In conclusion, 62 patients with chILD were analyzed in this study. Genetic variants considered causative of lung dysfunction were detected in 11 of the 62 patients (18\%), consisting of SFTPC mutations in six, NKX2.1 mutations in three, and FOXF1 mutations in two cases. Among Japanese chILD patients, NKX2.1 and SFPTC variants appeared to be more common than $A B C A 3$ variants. We are now planning to establish gene panel for chILD using next-generation sequencer and whole-exome analysis for undiagnosed cases.

\section{ACKNOWLEDGMENTS}

We thank all the neonatologists and pediatricians who provided information and materials used in this study.

\section{STATEMENT OF FINANCIAL SUPPORT}

This research was supported by the "Practical Research Project for Rare/ Intractable Diseases" from Japan Agency for Medical Research and Development, AMED, and by the Morinaga Foundation for Health and Nutrition.

Disclosure: The authors declare no conflict of interest.

\section{REFERENCES}

1. Spagnolo P, Bush A.. Interstitial lung disease in children younger than 2 years. Pediatrics 2016;137:e20152725. 


\section{Genetic basis for chILD $\quad$ Articles}

2. Akin MR, Nguyen GK.. Pulmonary alveolar proteinosis. Pathol Res Pract 2004;200:693-8 discussion 699-700.

3. Janney CG, Askin FB, Kuhn C 3rd. Congenital alveolar capillary dysplasia -an unusual cause of respiratory distress in the newborn. Am J Clin Pathol 1981;76:722-.

4. Koh DM, Hansell DM.. Computed tomography of diffuse interstitial lung disease in children. Clin Radiol 2000;55:659-7.

5. Hartl D, Griese M.. Interstitial lung disease in children-genetic background and associated phenotypes. Respir Res 2005;6:32.

6. Nogee LM, de Mello DE, Dehner LP, Colten HR. Brief report: deficiency of pulmonary surfactant protein $\mathrm{B}$ in congenital alveolar proteinosis. $\mathrm{N}$ Engl J Med 1993;328:406-10.

7. Nogee LM, Dunbar AE 3rd, Wert SE, Askin F, Hamvas A, Whitsett JA.. A mutation in the surfactant protein $\mathrm{C}$ gene associated with familial interstitial lung disease. N Engl J Med 2001;344:573-9.

8. Shulenin S, Nogee LM, Annilo T, Wert SE, Whitsett JA, Dean M.. ABCA3 gene mutations in newborns with fatal surfactant deficiency. $\mathrm{N}$ Engl J Med 2004;350:1296-303.

9. Hamvas A, Deterding RR, Wert SE, et al. Heterogeneous pulmonary phenotypes associated with mutations in the thyroid transcription factor gene NKX2-1. Chest 2013;144:794-804.

10. Stankiewicz P, Sen P, Bhatt SS, et al. Genomic and genic deletions of the FOX gene cluster on 16q24.1 and inactivating mutations of FOXF1 cause alveolar capillary dysplasia and other malformations. Am J Hum Genet 2009;84:780-91.

11. Dirksen U, Nishinakamura R, Groneck P, et al. Human pulmonary alveolar proteinosis associated with a defect in GM-CSF/IL-3/IL-5 receptor common beta chain expression. J Clin Invest 1997;100:2211-7.

12. Suzuki T, Sakagami T, Rubin BK, et al. Familial pulmonary alveolar proteinosis caused by mutations in CSF2RA. J Exp Med 2008;205: 2703-10.

13. Kitamura T, Tanaka N, Watanabe J, et al. Idiopathic pulmonary alveolar proteinosis as an autoimmune disease with neutralizing antibody against granulocyte/macrophage colony-stimulating factor. J Exp Med 1999;190: $875-0$.

14. Akimoto T, Cho K, Hayasaka I, et al. Hereditary interstitial lung diseases manifesting in early childhood in Japan. Pediatr Res 2014;76:453-8.

15. Uchida K, Nakata K, Carey B, et al. Standardized serum GM-CSF autoantibody testing for the routine clinical diagnosis of autoimmune pulmonary alveolar proteinosis. J Immunol Methods 2014;402:57-70.

16. Brasch $F$, Griese $M$, Tredano $M$, et al. Interstitial lung disease in a baby with a de novo mutation in the SFTPC gene. Eur Respir J 2004;24:30-9.

17. Poterjoy BS, Vibert Y, Sola-Visner M, McGowan J, Visner G, Nogee LM.. Neonatal respiratory failure due to a novel mutation in the surfactant protein C gene. J Perinatol 2010;30:151-3.

18. Sen P, Yang Y, Navarro C, et al. Novel FOXF1 mutations in sporadic and familial cases of alveolar capillary dysplasia with misaligned pulmonary veins imply a role for its DNA binding domain. Hum Mutat 2013;34:801-11.

19. Bonella F, Ohshimo S, Miaotian C, Griese M, Guzman J, Costabel U.. Serum KL-6 is a predictor of outcome in pulmonary alveolar proteinosis. Orphanet J Rare Dis 2013;8:53.

20. Guillot L, Epaud R, Thouvenin G, et al. New surfactant protein C gene mutations associated with diffuse lung disease. J Med Genet 2009;46: $490-4$.
21. Garmany TH, Wambach JA, Heins HB, et al. Population and diseasebased prevalence of the common mutations associated with surfactant deficiency. Pediatr Res 2008;63:645-9.

22. Salerno T, Peca D, Menchini L, et al. Surfactant protein C-associated interstitial lung disease; three different phenotypes of the same SFTPC mutation. Ital J Pediatr 2016;42:23.

23. Ahmed S, Ackerman V, Faught P, Langston C.. Profound hypoxemia and pulmonary hypertension in a 7-month-old infant: late presentation of alveolar capillary dysplasia. Pediatr Crit Care Med 2008;9:e43-6.

24. Ito $\mathrm{Y}$, Akimoto $\mathrm{T}$, Cho $\mathrm{K}$, et al. A late presenter and long-term survivor of alveolar capillary dysplasia with misalignment of the pulmonary veins. Eur J Pediatr 2015;174:1123-6.

25. Szafranski P, Gambin T, Dharmadhikari AV, et al. Pathogenetics of alveolar capillary dysplasia with misalignment of pulmonary veins. Hum Genet 2016;135:569-86.

26. Lazzaro D, Price M, de Felice M, Di Lauro R. The transcription factor TTF-1 is expressed at the onset of thyroid and lung morphogenesis and in restricted regions of the foetal brain. Development 1991;113:1093-4.

27. Devriendt K, Vanhole C, Matthijs G, de Zegher F. Deletion of thyroid transcription factor-1 gene in an infant with neonatal thyroid dysfunction and respiratory failure. N Engl J Med 1998;338:1317-8.

28. Peall KJ, Kurian MA.. Benign hereditary chorea: an update. Tremor Other Hyperkinet Mov 2015;14:314.

29. Carré A, Szinnai G, Castanet M, et al. Five new TTF1/NKX2.1 mutations in brain-lung-thyroid syndrome: rescue by PAX8 synergism in one case. Hum Mol Genet 2009;18:2266-76.

30. Iwatani N, Mabe H, Devriendt K, Kodama M, Miike T.. Deletion of NKX2.1 gene encoding thyroid transcription factor-1 in two siblings with hypothyroidism and respiratory failure. J Pediatr 2000;137:272-6.

31. Nagasaki K, Narumi S, Asami T, Kikuchi T, Hasegawa T, Uchiyama M.. Mutation of a gene for thyroid transcription factor-1 (TITF1) in a patient with clinical features of resistance to thyrotropin. Endocr J 2008;55:875-8.

32. Narumi S, Muroya K, Asakura Y, Adachi M, Hasegawa T.. Transcription factor mutations and congenital hypothyroidism: systematic genetic screening of a population-based cohort of Japanese patients. J Clin Endocrinol Metab 2010;95:1981-5.

33. Ito K, Iwata S, Nakashima Y, Suzuki E, Hasegawa Y, Taketani T. Father and daughter cases with benign hereditary chorea, hypothyroidism, and recurrent pulmonary infections having TTF1/NKX2-1 gene mutations (In Japanese). J Jpn Pediatr Soc 2011;115:113-7.

34. Uematsu M, Haginoya K, Kikuchi A, et al. Hypoperfusion in caudate nuclei in patients with brain-lung-thyroid syndrome. J Neurol Sci 2012;315:77-81.

35. Konishi T, Kono S, Fujimoto M, et al. Benign hereditary chorea: dopaminergic brain imaging in patients with a novel intronic NKX2.1 gene mutation. J Neurol 2013;260:207-13.

36. Hayashi S, Yagi M, Morisaki I, Inazawa J.. Identical deletion at $14 \mathrm{q} 13.3$ including PAX9 and NKX2-1 in siblings from mosaicism of unaffected parent. J Hum Genet 2015;60:203-6.

37. Gower WA, Nogee LM.. Surfactant dysfunction. Paediatr Respir Rev 2011;12:223-9.

38. Turcu S, Ashton E, Jenkins L, Gupta A, Mok Q.. Genetic testing in children with surfactant dysfunction. Arch Dis Child 2013;98:490-5. 